\title{
Gründung des Dachverbandes Deutschsprachiger PsychosenPsychotherapie (DDPP)
}

\author{
Dorothea von Haebler (Berlin) und Günter Lempa (München)
}

\section{Historischer Hintergrund}

Deutschland und Österreich haben in Bezug auf die Psychotherapie von Psychosen eine schwerwiegende Geschichte. Nach dem massenhaften Mord an den Erkrankten während der nationalsozialistischen Diktatur war die deutsche Universitätspsychiatrie noch lange nach dem zweiten Weltkrieg von der Endogenität der Psychosen, das heißt von weitgehend eigengesetzlich ablaufenden Prozesserkrankungen, bei denen Umweltfaktoren nur bei der Ausgestaltung der Symptomatik eine Rolle spielen, überzeugt. Das dann erst viel später akzeptierte biopsychosoziale Paradigma führte zunächst zu keinerlei Veränderung in der psychiatrischen Versorgung. Die Behandlung psychotischer Patienten orientiert sich weitgehend am Modell einer Störung des Gehirnstoffwechsels, wobei flankierende oder zusätzliche sozialpsychiatrische Maßnahmen dem Management einer pharmakologisch nicht beeinflussbaren Chronifizierung entsprechen. Diese Situation in der psychiatrischen Versorgung stellt den historischen Hintergrund für die schließlich erfolgte Gründung des Dachverbandes Deutschsprachiger PsychosenPsychotherapie (DDPP) dar.

\section{Gründungsprozess}

Der Ursprung des DDPP reicht einige Jahrzehnte zurück. Damals entstand bei Psychoanalytikern und tiefenpsychologischen Psychotherapeuten, die mit psychotischen Menschen arbeiteten, das Bedürfnis, ihrem therapeutischen Ansatz in der Universitäts- und Richtlinienpsychiatrie zu einer größeren Bedeutung zu verhelfen. Auslöser für die Aktivitäten waren von dem eigenen Fachverband (DGPPN) ${ }^{1}$ konsequent abgelehnte Beiträge für Kongresse. Dagegen standen die klinischen Erfahrungen von psychodynamisch arbeitenden Therapeuten, die durchaus positive Behandlungsergebnissee mit einer Methode erzielten, die offiziell, nach den geltenden Behandlungsleitlinien, aufgrund der Studienlage keineswegs empfohlen wurde. So war es zur Gründung mehrerer Initiativen, die sich mit der Therapie und Praxis der Psychosenpsychotherapie befassten, gekommen. In Deutschland ist dies die Überregionale Weiterbildung in analytischer Psychosentherapie in 
München, das Frankfurter Psychoseprojekt (ebenfalls analytisch), der Arbeitskreis Psychotherapie der Psychosen in Tiefenbrunn bei Göttingen, die norddeutsche Arbeitsgemeinschaft für Psychodynamische Psychiatrie in Hamburg und schliesslich das methodenoffene überregionale Symposium für Psychosenpsychotherapie an der Charité Campus Mitte in Berlin. In diesen Initiativen wurden seit etwa 20 Jahren neue Konzepte der Theorie und Behandlungstechnik entwickelt. In einer längeren Vorbereitungsphase, die zuerst psychoanalytisch dominiert war, bildete sich dann auch mit erheblich kontroversen Diskussionen und Auseinandersetzungen bei der Mehrzahl der Beteiligten die Überzeugung, dass ein Zusammenschluss im Sinne eines schulen- und methodenoffenen Dachverbandes aller Therapeuten, die mit psychotischen Patienten arbeiten, die aussichtsreichste und stimmigste Lösung darstelle, um das angestrebte Ziel zu erreichen. Dieses besteht in erster Linie darin, für psychotische Patienten in einem größeren Ausmass, als dies bisher in der psychiatrischen Versorgung möglich ist, qualifizierte psychotherapeutische Behandlungen verfügbar zu machen und diese zu einem Selbstverständnis im Angebot werden zu lassen. Neben der durch einen methodenoffenen Verband gesteigerten Möglichkeit, auch gesundheitspolitisch aktiv werden zu können, erschien vielen auch die Überlegung zutreffend, dass sich in der bisherigen, nur wenig erfolgreichen Kommunikation verschiedener Paradigmen (und Therapeuten) in der Psychiatrie teilweise auch psychotische Mechanismen (Ängste vor Identitätsverlust, Idealisierung und Entwertung) widerspiegeln. Insofern erschien es ein erfolgversprechender Ansatz, einen berufsgruppen- und verfahrensübergreifenden Dialog und Austausch innerhalb des DDPP zu etablieren, was am ehesten der Versorgungsrealität vieler Patienten mit psychotischen Störungen entspricht, die oft von multiprofessionellen Teams bzw. von Therapeuten mit unterschiedlichem therapeutischen Ansatz behandelt werden.

Der Einladung zur Gründung des DDPP an Therapeuten verschiedener Verfahren folgten viele Kollegen, Institutionen, Kammern und Verbände. Sie zeigten ein ungeahntes Interesse. Unabhängig von einer psychotherapeutischen Schule und über die verschiedenen Berufsgruppen hinweg bestand ein Konsens in den Zielen. Es hatte bereits mehrere die Dachverbandsgründung vorbereitende Treffen an der Charité in Berlin gegeben, bei welchen eine regelrechte Aufbruchsstimmung entstanden war, die sich schliesslich auch in dem sehr gut besuchten Gründungskongress niederschlug. Verbände, Kammern, Ausbildungsinstitute, Kliniken und Einzelpersonen vieler therapeutischer Richtungen beteiligten sich personell und auch finanziell durch ihre Spende bei der Gründung des DDPP, die dann auf dem 
Gründungskongress an der Charité Campus Mitte in Berlin vom 6.-8. Mai 2011 stattfand.

\section{Arbeitsgruppen}

Der Dachverband hat drei Arbeitsgruppen eingerichtet, die sich mehrmals im Jahr treffen und sich mit den Themen Vernetzung, Forschung sowie Aus-, Fort- und Weiterbildung befassen. In der Arbeitsgruppe Vernetzung werden die mangelnde Kooperation und die institutionell und finanzierungspolitisch bedingten Gräben zwischen stationärer und ambulanter Behandlung thematisiert. Ausserdem werden Strukturen erarbeitet, die praktizierende Psychosetherapeuten bei der Arbeit mit auch schwer und komplex psychisch Kranken entlasten und unterstützen können. Die Aus-, Fort- und Weiterbildung findet in der Psychiatrie zu einseitig statt. Bei dem Ziel einer qualifizierten Psychotherapie für psychotisch erkrankte Menschen werden dringend besser ausgebildete Psychosepsychotherapeuten benötigt. Ein Ziel des DDPP und Inhalt dieser Arbeitsgemeinschaft ist es somit auch, neben den in den oben angeführten Verbänden stattfindenden Fortbildungen eigene Ausbildungsprogramme anzubieten. Hier ist zeitgleich mit der DDPP Gründung ein weiterbildender Masterstudiengang «IntegrierteVersorgung psychotisch erkrankter Menschen» entstanden, der als ein Werkzeug zur qualifizierten berufsgruppenübergreifenden und verfahrensvielfältigen Weiterbildung in der Psychosentherapie verstanden wird (siehe Anhang). Die Arbeitsgemeinschaft Forschung ist im Moment noch mit einer Bestandsaufnahme von aktuellen Forschungsaktivitäten zur Psychosenpsychotherapie und bereits existierenden Ergebnissen beschäftigt. Kognitiv- und verhaltenstherapeutische Psychosentherapien sind vielfach untersucht, während die tiefenpsychologischen und analytischen Therapien zwar häufig praktiziert werden, nach den Cochrane-Richtlinien, die in der offiziellen Psychiatrie als gültig angesehen werden, aber keine ausreichende Evidenz ihrer Wirksamkeit nachweisen können. Hier bietet die Forschungsgruppe ein großes Potential, methodenvergleichende und multizentrische Forschung zu konzipieren und durchzuführen.

Aus den Arbeitsgruppen sollen sich Vorschläge für die Verbesserung der Versorgung von Menschen mit Psychosen ergeben, um deren konkrete Umsetzung der Verband sich dann bemühen wird.

Auch die gesundheitspolitischen Aktivitäten des Dachverbands erscheinen aktuell wichtig, weil sich derzeit eine grundlegende Umstrukturierung der psychiatrischen Versorgung abzeichnet (Stichwort: «integrierte Versorgung»). Dabei droht die Gefahr einer rein ökonomisch ausgerichteten Behandlung von 
Menschen, die unter Psychosen leiden. Genau mit dieser Problematik hat sich der zweite Kongress mit dem Thema «Psychotherapie für alle Psychosen. Möglichkeiten und Grenzen» des DDPP befasst. Die DDPP Kongresse bieten die Gelegenheit, Perspektivenreichtum in den Vorträgen, aber auch in der konzentrierten Fallarbeit zu erleben und eigene Expertise auch in den Arbeitsgruppen einzubringen.

Die Idee des Dachverbandes DDPP brachte schon vor seiner Gründung viele Menschen verschiedener Professionen und Methoden zusammen. Es besteht ein Konsens über das Selbstverständnis, dass Psychosekranken eine qualifizierte Psychotherapie zugänglich sein muss. Die Wege dorthin sollen im DDPP gemeinsam erdacht, strukturiert, durch- und umgesetzt werden.

Informationen zum DDPP: www.ddpp.eu.

\section{Anhang}

Masterstudiengang «Integrierte Versorgung psychotisch erkrankter Menschen»

Ein oben bereits erwähntes Instrument, um qualifiziertere Therapeuten auszubilden, die sich dann in leitender Position effektiv und ressourcensparend in den Versorgungsstrukturen einbringen können, ist der Masterstudiengang «Integrierte Versorgung psychotisch erkrankter Menschen» der im WS 2012/2013 in Berlin starten wird.

\section{Idee}

Das Verständnis der Ursachen psychotischer Erkrankungen wurde in den letzten 40 Jahren erheblich differenzierter. Verschiedene qualifizierte Behandlungsmethoden stehen mittlerweile zur Verfügung. Die herkömmlichen Behandlungsmodelle werden dieser Komplexität jedoch nicht mehr gerecht, insbesondere in Anbetracht der sich zunehmend abzeichnenden grundlegenden Umstrukturierung in der psychiatrischen Versorgung. Es wird in der Zukunft entscheidend wichtig sein, über eine professionelle Expertise für die interdisziplinäre Zusammenarbeit im Team und zwischen dem stationären, komplementären und ambulanten Bereich zu verfügen. Eine erheblich größere Bedeutung als bisher wird auch der Trialog, die Zusammenarbeit mit den Betroffenen und ihren Verbänden einnehmen. Für diese Aufgabe existieren derzeit keine ausreichend ausgebildeten professionellen Experten. Der neue Masterstudiengang wird genau an dieser Stelle ansetzen. Im Bereich der Psychosenpsychotherapie soll Wissen und Handlungskompetenz unterschiedlicher Disziplinen und Methoden zusammengeführt und aufeinander bezogen werden. 


\section{Form}

Eine methoden- und berufsgruppenübergreifende Weiterbildung wird durch die enge Kooperation von vier Hochschulen aus Berlin und Hamburg mit je eigener Expertise ermöglicht: der Charité, Universitätsmedizin Berlin, dem Universitätsklinikum Eppendorf (UKE), der Katholischen Hochschule für Sozialwesen Berlin (KHSB) und der ausrichtenden International Psychoanalytic University (IPU) Berlin. Das Studium steht Absolventen eines berufsqualifizierenden Hochschulstudiums (z. B. Medizin, Psychologie, Soziale Arbeit, Heilpädagogik usw.) mit mindestens einjähriger, einschlägiger Berufserfahrung offen; es dauert sechs Semester und ist berufsbegleitend. Im Beruf Stehende können sich auf universitärem Niveau weiterbilden und so Theorie und Praxis verbinden.

\section{Inhalt}

Im Zentrum dieses Studienganges steht eine wissenschaftliche Auseinandersetzung mit dem Phänomen Psychose. Die professionelle, die gesellschaftliche und die subjektive Perspektive darauf werden vermittelt und ausdifferenziert. Therapeutische Haltung, Kompetenz und Authentizität werden durch ein über das gesamte Studium verlaufendes Modul mit supervidierter Fallarbeit im Team vermittelt. Leitung, Führung und Forschungsmodule ermöglichen es den Absolventen dieses Studienganges, nach Abschluss eine effektivere Gestaltung einer integrierten und qualifizierten Versorgung psychotisch erkrankter Menschen innerhalb des Versorgungssystems umzusetzen. Sie lernen, die berufsgruppenspezifischen Handlungsaufträge und Expertisen aufeinander zu beziehen und diese strukturell umzusetzen. Der aktuelle Wissensstand zum Phänomen Psychose wird methodenübergreifend vertieft und Kompetenzen zu bedürfnisangepasster, effektiver und gleichzeitig ressourcensparender Zusammenarbeit innerhalb des psychiatrischen Versorgungssystems vermittelt.

\section{Ziele}

Die Absolventen dieses Studienganges werden befähigt, als Ärzte, Psychotherapeuten oder Sozialarbeiter mit den unterschiedlichen Berufsgruppen des Gesundheits- und Sozialwesens zu kooperieren. Sie spezialisieren sich auf die Behandlung und Begleitung psychisch auch schwer und chronisch erkrankter Menschen und vertiefen die Fähigkeit, mit interdisziplinären Teams, Betroffenen und Angehörigen zu arbeiten. Sie lernen, integrative und innovative Versorgungsund Finanzierungsmodelle weiter zu entwickeln und umzusetzen. Dadurch gewinnen sie die Fähigkeit, Leitungspositionen in den Versorgungssystemen auszufüllen. 
Informationen zum Studiengang: www.ipu-berlin.de.

\section{Anmerkung}

1 Deutsche Gesellschaft für Psychiatrie, Psychotherapie und Nervenheilkunde. 